\title{
Upregulation of TRAF-3 by shear stress blocks CD40-mediated endothelial activation
}

\author{
Carmen Urbich, ${ }^{1}$ Ziad Mallat, ${ }^{2}$ Alain Tedgui, ${ }^{2}$ Matthias Clauss, ${ }^{3}$ Andreas M. Zeiher, ${ }^{1}$ \\ and Stefanie Dimmeler ${ }^{1}$
}

${ }^{1}$ Molecular Cardiology, Department of Internal Medicine IV, University of Frankfurt, Frankfurt, Germany ${ }^{2}$ Hôpital Lariboisiere, INSERM U141, Paris, France

${ }^{3}$ Max-Planck Institut for Vascular Biology, Bad Nauheim, Germany

Address correspondence to: Stefanie Dimmeler, Molecular Cardiology, Department of Internal Medicine IV, University of Frankfurt, Theodor Stern-Kai 7, 60590 Frankfurt, Germany.

Phone: 49-69-6301-7440; Fax: 49-69-6301-7113; E-mail: Dimmeler@em.uni-frankfurt.de.

Received for publication June 26, 2001, and accepted in revised form September 24, 2001.

\begin{abstract}
Atherosclerosis is an inflammatory disease of large arteries that is initiated through the activation of endothelium by proinflammatory mediators. CD40 receptor stimulation has been implicated in the pathogenesis of atherosclerosis. One of the most important atheroprotective stimuli is the viscous drag (shear stress) generated by the streaming blood acting on the endothelial monolayer. Here, we demonstrate that shear stress prevents CD40 ligand-induced endothelial cell activation, and we identify upregulation of TNF receptor-associated factor-3 (TRAF-3) as a potent CD40-inhibitory mechanism. Shear stress specifically upregulates TRAF-3 in cultured endothelial cells. Moreover, in the endothelial cells overlying human atherosclerotic plaques, TRAF-3 expression is upregulated in areas with high shear stress. Overexpression of TRAF-3 inhibits endothelial expression of proinflammatory cytokines and tissue factor and blocks DNA-binding activity of the transcription factor AP-1; it thereby prevents CD40-induced endothelial activation. Thus, upregulation of TRAF-3 represents a novel mechanism for preserving the functional integrity of the endothelial monolayer.
\end{abstract}

J. Clin. Invest. 108:1451-1458 (2001). DOI:10.1172/JCI200113620.

\section{Introduction}

Phenotypic modulation of the endothelium to an activated state contributes to the pathogenesis of cardiovascular diseases such as atherosclerosis $(1,2)$. Ligation of endothelial CD40 by CD40 ligand (CD40L), either expressed on activated monocytes or T-cells, or disgorged from platelet granules after activation, produces various inflammatory cytokines $(3,4)$, chemokines such as monocyte chemoattractant protein-1 (MCP-1), procoagulant activity (5), adhesion molecules, matrix-degrading metalloproteinases (6), and inflammatory lipid mediators. All of these mediators have been implicated in the pathogenesis and progression of atherosclerosis $(7,8)$. Indeed, disruption of CD40-CD40L interaction in experimental models of atherosclerosis has been shown not only to inhibit lesion development $(9,10)$, but also to interrupt progression of lesions and to change their composition to a more stable, collagen-rich phenotype $(10,11)$.

The activation of proinflammatory and procoagulatory activity by CD40 receptor stimulation involves transcriptional events such as activation of NF- $\mathrm{KB}$ and AP-1 $(7,12)$. The proximal signal transduction in CD40mediated transcription factor activation depends on the family of TNF receptor-associated factors (TRAFs) (13). TRAF-2, TRAF-5, and TRAF- 6 mediate the CD40induced activation of the transcription factors, whereas TRAF-3 blocks CD40-induced signaling (13-15).
The viscous drag (shear stress) provided by flowing blood exerts a potent atheroprotective effect (16). This is evidenced by the finding that atherosclerotic lesions preferentially develop in areas with low or turbulent flow, whereas regions with uniform laminar flow are protected $(2,17)$. The endothelial lining is the primary sensor of wall shear stresses, and functions as a transducer of these biomechanical stimuli into biological responses within the vessel wall $(2,18,19)$. Therefore, we hypothesized that shear stress might interfere with CD40-mediated endothelial cell activation. We demonstrate that shear stress blocks the proinflammatory and procoagulatory activity of CD40L. Because CD40 signaling is regulated by TRAFs, we further investigated the influence of shear stress on TRAF proteins. We identified TRAF-3 upregulation as an inhibitory mechanism that abrogates CD40 signaling in endothelial cells.

\section{Methods}

Cell culture, Western blot analysis, and immunoprecipitation. Pooled human umbilical venous endothelial cells (HUVECs) and human cardiac microvascular endothelial cells were purchased from Clonetics (Solingen, Germany), and were cultured as previously described (20). Shear stress was applied by a cone-and-plate apparatus with 15 dynes $/ \mathrm{cm}^{2}$ (20). Recombinant CD40L was kindly donated by Immunex Corp. (Seat- 
tle, Washington, USA). Nordihydroguaiaretic acid (NDGA), pyrolidine dithiocarbamate (PDTC), and curcumin were purchased from Sigma-Aldrich (Taufkirchen, Germany). IL-8 release was detected in cell culture supernatants by Quantikine immunoassay (R\&D Systems Inc., Wiesbaden, Germany). Tissue factor activity was measured with a clotting assay as described previously (21).

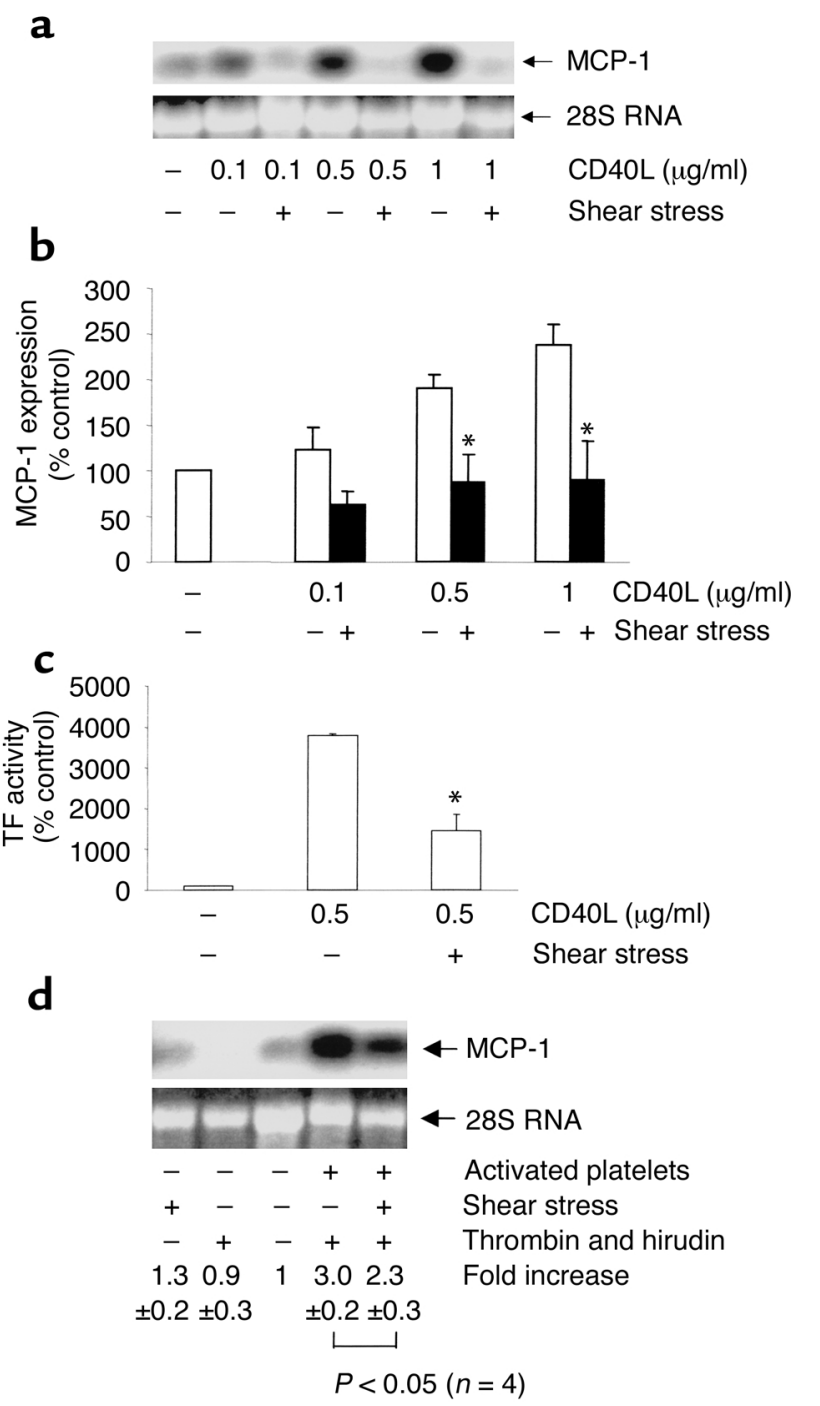

\section{Figure 1}

Shear stress prevents CD40L-induced endothelial cell activation. HUVECs were preexposed to shear stress for 12 hours before stimulation with recombinant $C D 40 \mathrm{~L}$ for 6 hours under continuous flow conditions. MCP-1 mRNA expression was monitored by Northern blot analysis as representatively shown (a). The quantification is shown in (b); data are mean \pm SEM. $n=4,{ }^{*} P<0.05$ vs. CD40L stimulation. (c) Shear stress inhibits CD40-induced tissue factor activity. After preexposure of HUVECs to shear stress, tissue factor activity was detected using a clotting assay. Data are mean \pm SEM. $n=4$, ${ }^{*} P<0.05$ vs. CD40L stimulation. (d) Platelets were activated as described in Methods and incubated with HUVECs that had been preexposed to shear stress for 12 hours. MCP-1 expression was detected after 6 hours of coincubation. A representative Northern blot is shown. Quantification was performed with four experiments. $P<0.05$ vs. activated platelets. TF, tissue factor.
For Western blotting, HUVEC homogenates were obtained as described previously (20), and blots were incubated with the following antibodies: TRAF-2, TRAF-3, TRAF-5, CD40, IкB $\alpha$, topoisomerase I (Santa Cruz Biotechnology Inc., Santa Cruz, California, USA), actin (Chemicon International, Hofheim, Germany), ERK1/2, phospho-ERK1/2, phospho-JNK (Cell Signaling Technology Inc., Beverly, Massachusetts, USA), and phosphoJun (Upstate Biotechnology Inc., Lake Placid, New York, USA). To immunoprecipitate the CD40 receptor, cells were incubated overnight at $4^{\circ} \mathrm{C}$ with an antibody against CD40 (Upstate Biotechnology Inc.). After incubation with protein A/G agarose beads $(30 \mu \mathrm{l} / \mathrm{mg}$ protein; Santa Cruz Biotechnology Inc.) for 1.5 hours at $4^{\circ} \mathrm{C}$, the protein complexes were washed and analyzed for TRAF- 3 binding by Western blot. For nuclear and cytoplasmic separation, Nuclear and Cytoplasmic Extraction Reagents were used according to the instructions of the manufacturer (Pierce Chemical Co., Rockford, Illinois, USA).

Northern blot analysis and RNase protection assay. Northern blots were performed as previously described (22). TRAF-3 mRNA expression was analyzed with the ribonuclease protection assay using the multiprobe template set hAPO-5 according to the instructions of the manufacturer (PharMingen, San Diego, California, USA).

Platelet preparation and activation. The platelet-rich plasma obtained from healthy human subjects was collected into an equal volume of acid-citrate-dextrose buffer ( $38 \mathrm{mM}$ citric acid, $75 \mathrm{mM}$ trisodium citrate, $124 \mathrm{mM}$ glucose; $\mathrm{pH} 4.5$ ) and centrifuged at $700 \mathrm{~g}$ for $10 \mathrm{~min}$ utes. Platelets were resuspended in Tyrode's/HEPES buffer $\left(1.8 \mathrm{mM} \mathrm{CaCl}_{2}, 2.7 \mathrm{mM} \mathrm{KCl}, 0.5 \mathrm{mM} \mathrm{MgCl}_{2}, 137\right.$ $\mathrm{mM} \mathrm{NaCl}, 10 \mathrm{mM}$ HEPES, $0.36 \mathrm{mM} \mathrm{NaH}_{2} \mathrm{PO}_{4}, 5 \mathrm{mM}$ glucose; pH 7.35), and centrifuged at $700 \mathrm{~g}$ for $10 \mathrm{~min}-$ utes. After resuspension, $1.5 \times 10^{8}$ platelets were incubated with HUVECs, and activated with $0.2 \mathrm{U} / \mathrm{ml}$ human thrombin (Boehringer Mannheim $\mathrm{GmbH}$, Mannheim, Germany). Cell culture dishes were centrifuged at $700 \mathrm{~g}$ for 2 minutes, and thrombin was neutralized after 5 minutes with $2 \mathrm{U} / \mathrm{ml}$ hirudin (Boehringer Mannheim GmbH).

Plasmid transfection. Plasmid encoding the human fulllength TRAF-3 was kindly donated by J. Reed (Burnham Institute, La Jolla, California, USA). The dominant-negative construct (amino acids 340-568) (23) was subcloned by PCR in PcDNA3.1-Myc-His (Invitrogen BV, Groningen, The Netherlands). Human full-length TRAF-2- and TRAF-5-encoding plasmids were kindly donated by S. Lederman (Department of Medicine, Columbia University, New York, New York, USA) (24), and were subcloned in pcDNA3.1-Myc-His. Clones with verified sequences were transfected in HUVECs $\left(3.5 \times 10^{5}\right.$ cells/6-cm well; $3 \mu \mathrm{g}$ plasmid DNA; $25 \mu$ l SuperFect) (Qiagen, Hilden, Germany), with a transfection efficiency of about $50 \%$, as previously described (20).

Transfection with decoy oligonucleotides. Phosphorothioate double-stranded decoy oligonucleotides $(1 \mu \mathrm{g})$ corresponding to the AP- 1 binding sequence ( $5^{\prime}$-AGCTTGTGAGTCAGAAGCT- $3^{\prime}$ ) or scrambled sequence (5'-TTGC- 

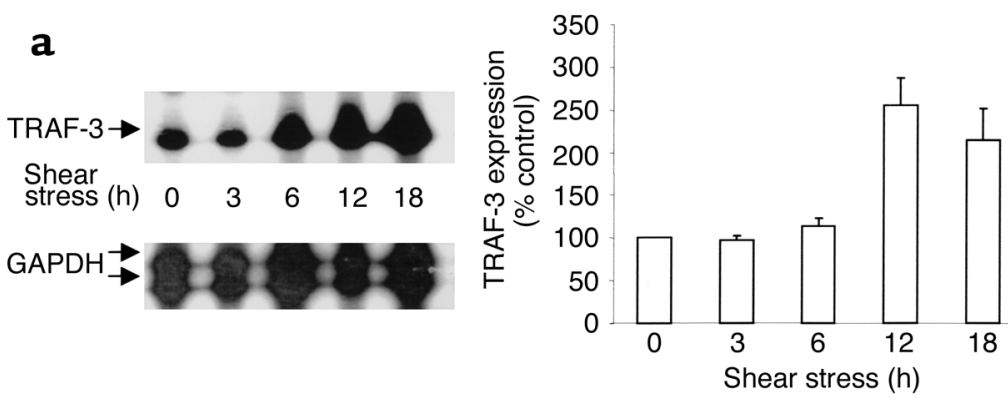

b Time dependency
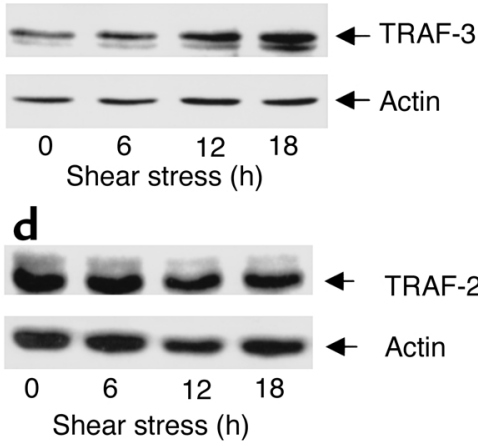

C Dose dependency
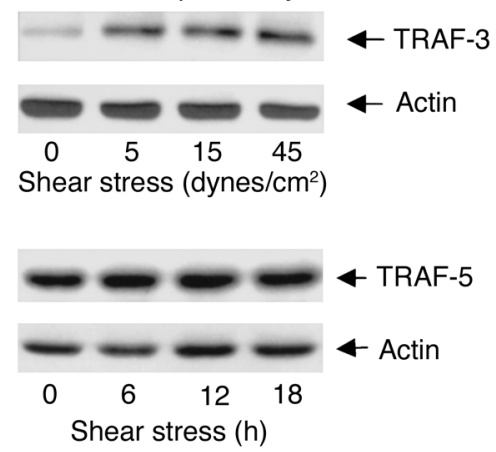
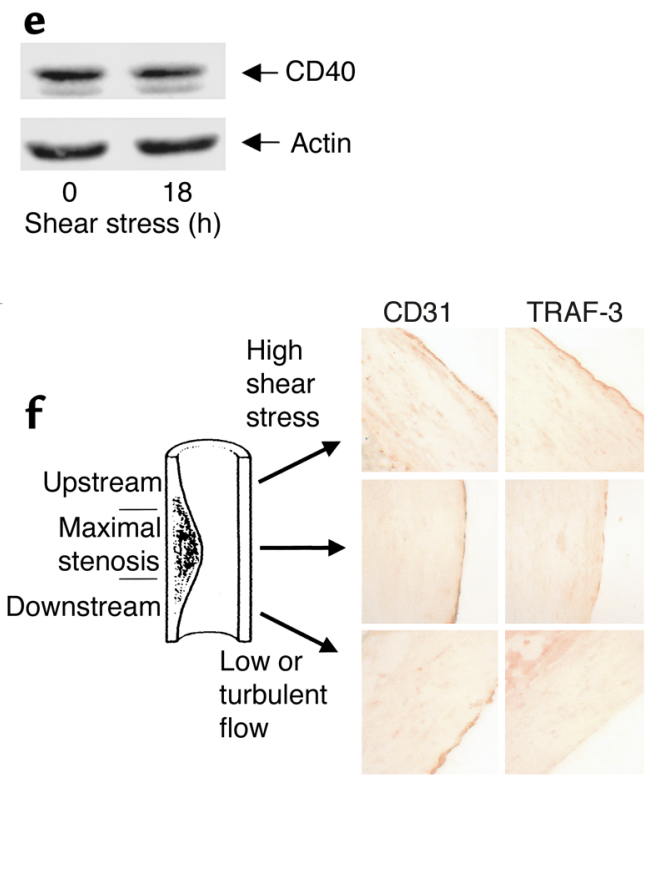

\section{Figure 2}

Shear stress upregulates TRAF-3 in vitro and in vivo. (a) HUVECs were exposed to shear stress, and TRAF-3 mRNA was detected by RNase protection assay, with GAPDH as loading control (mean \pm SEM; $n=3$ ). (b and $\mathbf{c}$ ) Time- and dose-dependent upregulation of TRAF-3 protein by shear stress exposure. HUVECs were exposed to shear stress as indicated and TRAF-3 protein was detected by Western blot analysis. Representative figures from three to four experiments are shown. (d and e) HUVECs were exposed to shear stress, and expression of TRAF-2, TRAF-5 (d), and CD40 receptor $(\mathbf{e})$ were detected by Western blot analysis $(n=3)$. (f) Human atherosclerotic plaques from nine patients with coronary artery disease were stained with TRAF-3 antibody (right), and endothelial cells were stained in serial sections with a CD31 antibody (left) to confirm cell type-specific expression. The scheme depicts different regions of the vessel (upstream and downstream areas of the plaques and in the maximal stenosis) with the local flow conditions. Representative sections are shown.

CGTACCTGACTTAGC-3') were incubated in $100 \mu \mathrm{l}$ RPMI medium in the presence of $5 \mu \mathrm{l}$ LipofectAMINE (Life Technologies Inc., Karlsruhe, Germany) for 30 minutes at room temperature. HUVECs $\left(4.0 \times 10^{5}\right.$ cells) were washed with RPMI and incubated with $2 \mathrm{ml}$ RPMI before adding the LipofectAMINE/oligonucleotide mixture. After 5 hours of further incubation, $3 \mathrm{ml}$ complete endothelial basal medium was added.

Immunostaining and immunobistochemistry. HUVECs were washed, fixed with $2 \%$ paraformaldehyde, and stained with antibodies against TRAF-2, TRAF-3, or TRAF-5 (1:50 in PBS/5\% FCS; Santa Cruz Biotechnology Inc.). Cells were incubated with a secondary anti-rabbit antibody linked to FITC (1:20 in PBS/5\% FCS; DAKO Corp., Hamburg, Germany), followed by counterstaining with 4',6-diamidino-2-phenylindole dihydrochloride (DAPI; $1 \mu \mathrm{g} / \mathrm{ml}$ ). Immunohistochemistry of human atherosclerotic plaques derived by endarterectomy from nine patients with coronary artery disease was performed with a TRAF-3 antibody (Santa Cruz Biotechnology Inc.). Semiquantitative examination was performed by two blinded investigators.

Electrophoretic mobility shift assay. Nuclear extracts from HUVECs were prepared with Nuclear and Cytoplasmic Extraction Reagents according to the manufacturer's instructions (Pierce Chemical Co., Rockford, Illinois,
USA). The double-stranded gel shift oligonucleotides (Santa Cruz Biotechnology Inc.) for AP-1 (5'CGCTTGATGACTCAGCCGGAA-3') were end-labeled with $\left[\gamma^{32} \mathrm{P}\right] A T P$. Nuclear extracts $(5 \mu \mathrm{g})$ were incubated in binding buffer with $1.5 \mu \mathrm{g}$ of poly(dI-dC), $1 \mathrm{mM}$ DTT, and ${ }^{32}$ P-labeled oligonucleotide probe $(0.5 \mathrm{ng})$ for 30 minutes at room temperature, and analyzed by electrophoretic mobility shift assay (EMSA) using a nondenaturing $5 \%$ polyacrylamide gel. The specificity of binding was examined by competition with unlabeled AP-1 and AP-1 mutant oligonucleotides (5'-CGCTTGATGACTTGGCCGGAA-3'), respectively. Supershift analysis was performed with $4 \mu \mathrm{g}$ of AP-1 Supershift antibody (Santa Cruz Biotechnology Inc.).

\section{Results}

Shear stress inbibits CD40-induced endothelial activation. To investigate the effect of shear stress on CD40-induced endothelial activation, HUVECs were exposed to shear stress before stimulation with recombinant CD40L. The proinflammatory response of endothelial cells was determined by measuring the expression of the proinflammatory chemoattractant protein MCP- 1 and the procoagulant tissue factor activity. As shown in Figure 1, a and b, preexposure to shear stress completely prevented CD40induced endothelial MCP-1 expression. Moreover, 
CD40L-induced tissue factor activity, which promotes thrombus formation, was significantly reduced by preexposure of the endothelial cells to laminar flow (Figure 1c).

Under pathophysiological conditions, activated platelets may serve as the stimulus for activation of the CD40 receptor in endothelial cells (25). Therefore, we performed analyses to determine whether shear stress may also protect against the proinflammatory effect induced by activated platelets. As illustrated in Figure 1d, activated platelets potently stimulated the expression of MCP-1, which was significantly reduced in HUVECs preexposed to shear stress (respective fold increase: $2.3 \pm 0.3$ vs. $3.0 \pm 0.2$, $P<0.05)$. The inhibitory effect of shear stress in this model is essentially identical to that achieved by neutralizing anti-CD40 antibodies (25). The lack of complete inhibition most likely reflects stimulation of various signaling pathways by activated platelets. A
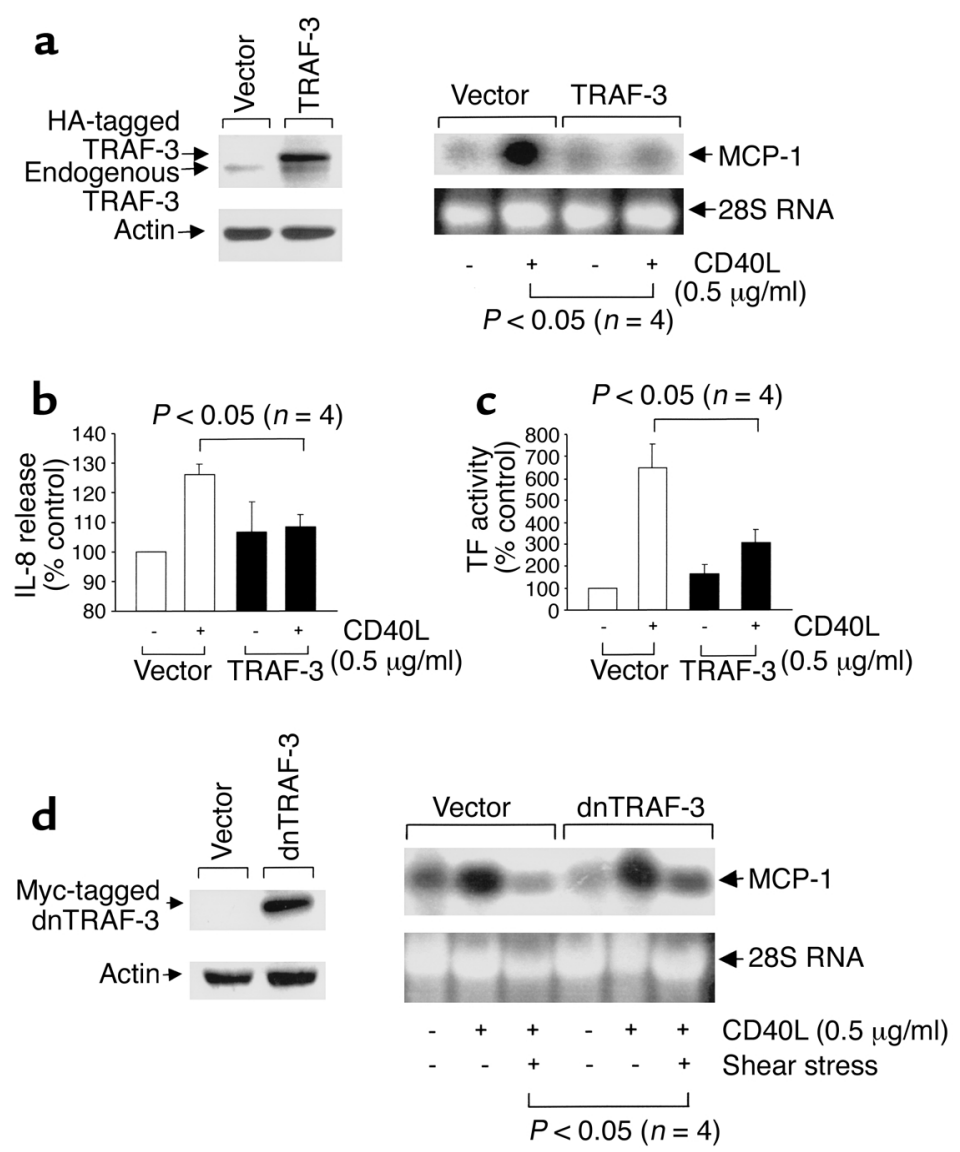

Figure 3

Role of TRAF-3 in CD40 signaling. (a-c) Effect of TRAF-3 overexpression on CD40L-stimulated MCP-1 expression (a, right), IL-8 release (b), and tissue factor activity (c). HUVECs were transfected with TRAF- 3 for 18 hours before stimulation of cells with CD40L for 6 hours. Expression of TRAF-3 is shown in a (left). Data are mean \pm SEM. (d) HUVECs were transfected with a dominantnegative TRAF-3 construct (dnTRAF3; amino acids 340-568) and preexposed to shear stress for 12 hours before stimulation with CD40L for an additional 6 hours under continuous application of shear stress. MCP-1 mRNA expression was detected by Northern blot. The expression of dnTRAF3 is shown in the left panel. MCP-1 expression in response to shear stress and/or CD40L is shown in the right panel. HA, hemagglutinin. similar inhibitory effect of shear stress was achieved when platelet-induced tissue factor expression was measured (data not shown). Taken together, these data show that shear stress exposure prevents CD40 signaling induced by either recombinant CD40L or by activated platelets, and thereby inhibits the proinflammatory and prothrombotic response of endothelial cells to CD40 activation.

Shear stress upregulates TRAF-3 expression in vitro and in vivo. Having demonstrated that shear stress prevents CD40L-induced endothelial cell activation, we attempted to characterize the underlying molecular mechanism. The intracellular signaling of CD40 is regulated by a family of cytoplasmic adapter proteins, TRAFs. Whereas most TRAFs, including TRAF-2 TRAF-5, promote intracellular signal transduction, TRAF- 3 inhibits CD40 signaling by an unknown mechanism $(14,15,26-28)$. Therefore, we examined the regulation of TRAF-2, TRAF- 3 , and TRAF-5 by shear stress. Shear stress timeand dose-dependently upregulates TRAF-3 mRNA and protein expression (Figure 2, a-c), whereas TRAF-2 and TRAF-5 protein expression remain unchanged upon application of shear stress (Figure 2d). Determination of TRAF-3 transcription by nuclear runon assays did not reveal any effect by shear stress (control: $104 \% \pm 14 \%$; shear stress: $114 \% \pm 18 \%$ compared with GAPDH).

Expression of the CD40 receptor was not altered by shear stress, excluding the possibility that shear stress inhibits CD40 signaling by receptor downregulation (Figure $2 \mathrm{e}$ ). These data suggest that shear stress interferes with CD40 signaling via specific upregulation of the inhibitory protein TRAF-3.

Next, we examined whether shear stress also affects TRAF-3 expression in vivo, by performing TRAF-3 immunostaining in human atherosclerotic plaques. As shown in Figure $2 \mathrm{f}$, endothelial TRAF-3 expression is specifically upregulated in the area upstream and within the maximal stenosis ("upstream part of the plaque"), which is characterized by high shear stress. In contrast, in areas distal to the maximal stenosis ("downstream part of the plaque"), where flow is unsteady or turbulent, only very weak staining or no staining was detected (Figure 2f). Semiquantitative grading of the immunohistochemical staining from 0 (absence of staining) to 4 (maximal staining) revealed significantly increased expression of TRAF-3 in the upstream area with high shear stress $(2.56 \pm 0.22)$ compared with the downstream part of the lesions $(1.4 \pm 0.4, n=9, P=0.0045)$. Taken together, our data illustrate that the level of shear stress determines the expression of TRAF-3 in endothelial cells in vitro and in vivo. 

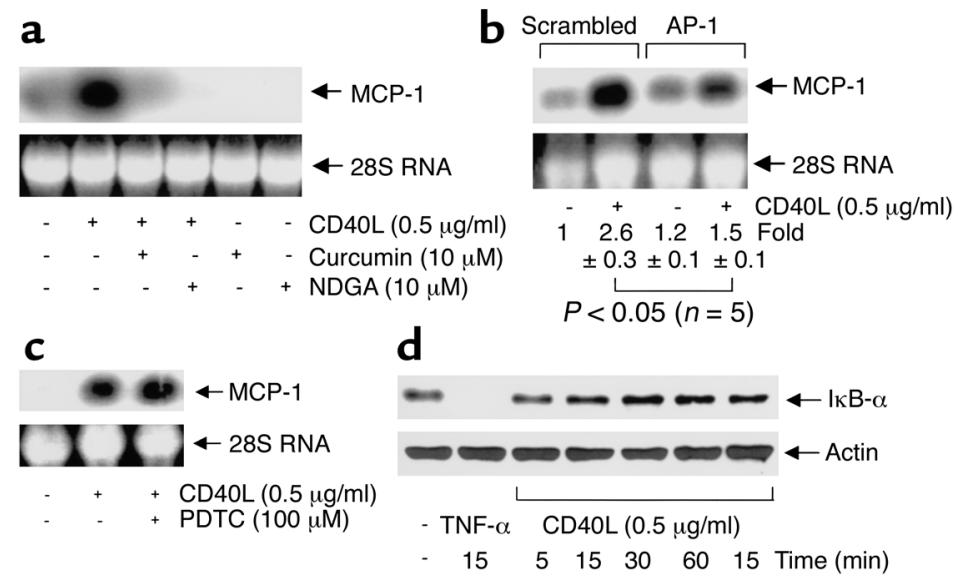

+ Shear stress $12 \mathrm{~h}$

\section{Figure 4}

CD40L-induced MCP-1 expression is mediated by AP-1. (a and c) HUVECs were preincubated for 30 minutes with PDTC, curcumin, or NDGA, then stimulated for 6 hours with CD40L. MCP-1 mRNA was detected by Northern blot analysis. 28S RNA served as loading control. Blots representative of three experiments are shown. (b) HUVECs were transfected with AP-1 decoy or scrambled oligonucleotides and stimulated with CD40L for 6 hours. MCP- 1 mRNA was detected by Northern blot analysis. 28S RNA served as loading control. A representative blot from five experiments is shown. (d) HUVECs were incubated with CD40L in the presence or absence of shear stress or TNF- $\alpha$ (as positive control). IKB- $\alpha$ protein was analyzed by Western blotting, with actin as loading control.
TRAF-3 blocks CD40-induced endothelial cell activation. In order to examine a causal role for TRAF-3 in modulation of endothelial cell activation, the effect of overexpressed TRAF-3 on CD40-stimulated endothelial cell activation was determined. TRAF-3 expression abrogated CD40-triggered MCP-1 expression and IL-8 release (Figure 3, a and b). Moreover, CD40L-stimulated tissue factor activity was significantly reduced by TRAF-3 overexpression (Figure 3c), clearly demonstrating that TRAF-3 is sufficient to inhibit CD40 signaling. To address the contribution of TRAF-3 to the inhibitory effect of shear stress, endothelial cells were transfected with a dominant-negative TRAF-3 construct (23). Dominant-negative TRAF-3 partially reversed the inhibitory effect of shear stress on CD40L-induced MCP-1 expression $(261 \% \pm 33 \%$ vs. $152 \% \pm 14 \%, n=4, P<0.05$; Figure $3 \mathrm{~d}$ ), thus demonstrating that shear stress requires TRAF- 3 upregulation to prevent CD40-induced endothelial activation. However, the incomplete inhibition suggests that shear stress exerts additional CD40 inhibitory effects that are independent of TRAF-3.

TRAF-3 inbibits AP-1 DNA binding. Next, we investigated the mechanism by which TRAF-3 interferes with CD40 signaling. Whereas expression of MCP-1 and proinflammatory cytokines was shown to be mainly attributed to activation of the transcription factors NF- $\kappa$ B and AP-1 (12), the distinct contribution of these transcription factors to CD40L-induced endothelial cell activation is less clear. Therefore, we first identified the transcription factor that mediates CD40L-induced MCP-1 expression in endothelial cells. As shown in Figure 4, CD40L-induced MCP-1 expression was blocked by pharmacological inhibitors of AP-1 such as NDGA or curcumin (Figure 4a), or by transfection of the cells with AP-1 decoy oligonucleotides (Figure 4b). In contrast, inhibition of NF- $\mathrm{KB}$ by PDTC did not affect CD40L-induced MCP-1 expression (Figure 4c). Moreover, CD40L stimulation did not alter the degradation of the NF- $\kappa \mathrm{B}$ inhibitor I $\kappa \mathrm{B} \alpha$ (Figure $4 \mathrm{~d})$. These data indicate that AP-1, but not NF- $\mathrm{B}$, mediates CD40Linduced MCP-1 expression in endothelial cells.
To elucidate the target of TRAF-3, we further investigated the effect of TRAF-3 expression on the downstream signaling pathway mediating CD40L-induced AP-1 activation. Activation of AP-1 requires homo- or heterodimer formation of Jun and Fos (29). Indeed, CD40L stimulated phosphorylation of JNK, the JNK substrate c-Jun, and ERK1/2 (Figure 5, a and b). However, TRAF-3 overexpression did not affect CD40Linduced activation of the JNK-ERK1/2 pathway (Figure 5 , a and $\mathrm{b}$ ). Consistent with this finding, coimmunoprecipitation studies revealed that TRAF-3 was not bound to the CD40 receptor (data not shown). Moreover, TRAF-3 was shown to localize mainly in the nuclei, as demonstrated by immunohistochemistry and biochemical fractionation, whereas the other members of the TRAF family such as TRAF- 2 and TRAF-5 revealed different localization (Figure 5, c and d). Similarly, predominant nuclear localization of TRAF-3 was also shown in human dermal and cardiac microvascular endothelial cells (Figure 5d and data not shown), but not in NIH 3T3 cells (data not shown). These data suggest that TRAF-3 does not interfere with the proximal signal events, but acts downstream of Jun activation. Therefore, we tested the effect of TRAF-3 on CD40L-induced AP-1 activation using EMSA. As illustrated in Figure 5e, TRAF-3 overexpression prevents CD40L-induced AP-1 activation. In contrast, overexpression of TRAF- 2 or TRAF-5 did not affect CD40L-stimulated AP-1 DNA binding activity (Figure 5e). Controls with unlabeled oligonucleotides and supershift in the presence of AP-1 antibody are shown in Figure 5f.

\section{Discussion}

Our data demonstrate that shear stress upregulates mRNA and protein expression of TRAF-3. The sustained upregulation of TRAF-3 in the endothelial monolayer that is exposed to laminar shear stress prevents the proinflammatory and procoagulatory activation of endothelial cells in response to CD40L. Compelling evidence suggests that CD40 is a major trigger eliciting a proinflammatory reaction on endothelial cells, and that it plays an important role in the formation of athero- 
a

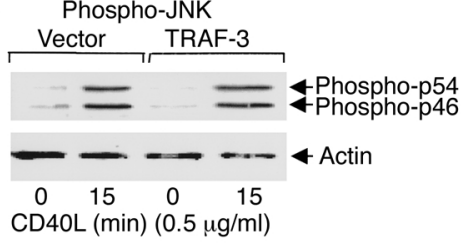

C
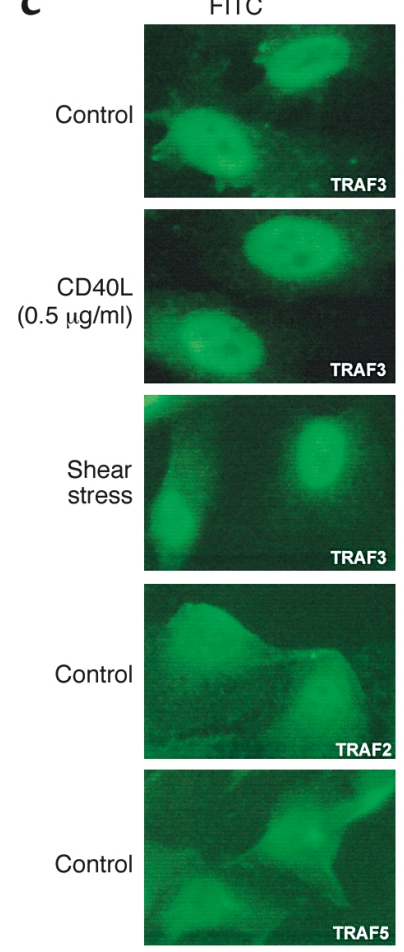

Phospho-C-Jun

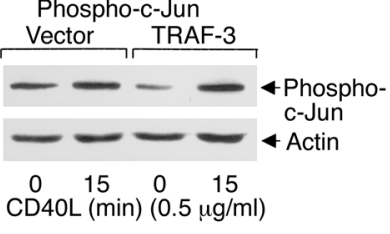

b
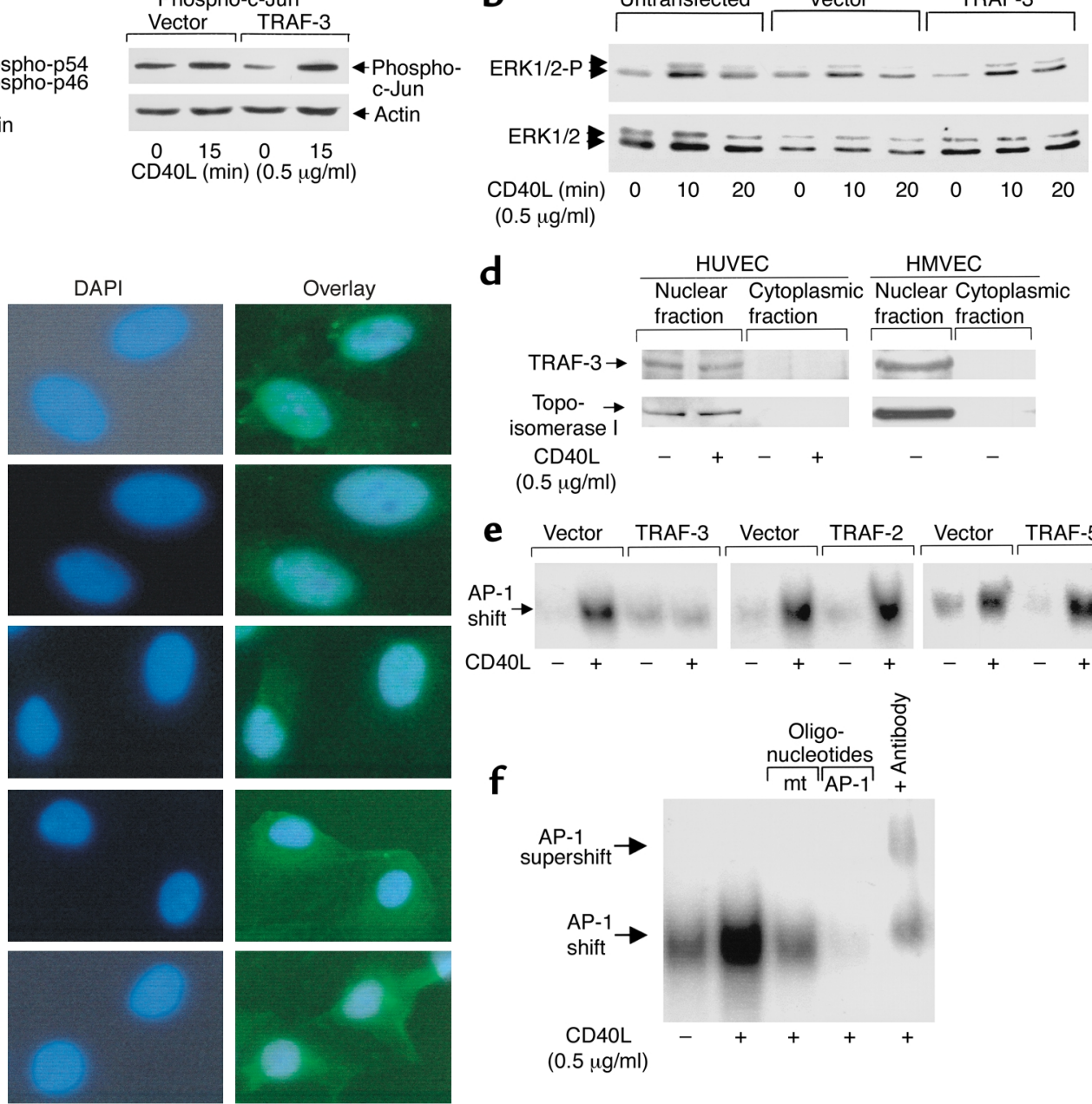

d HUVEC HMVEC
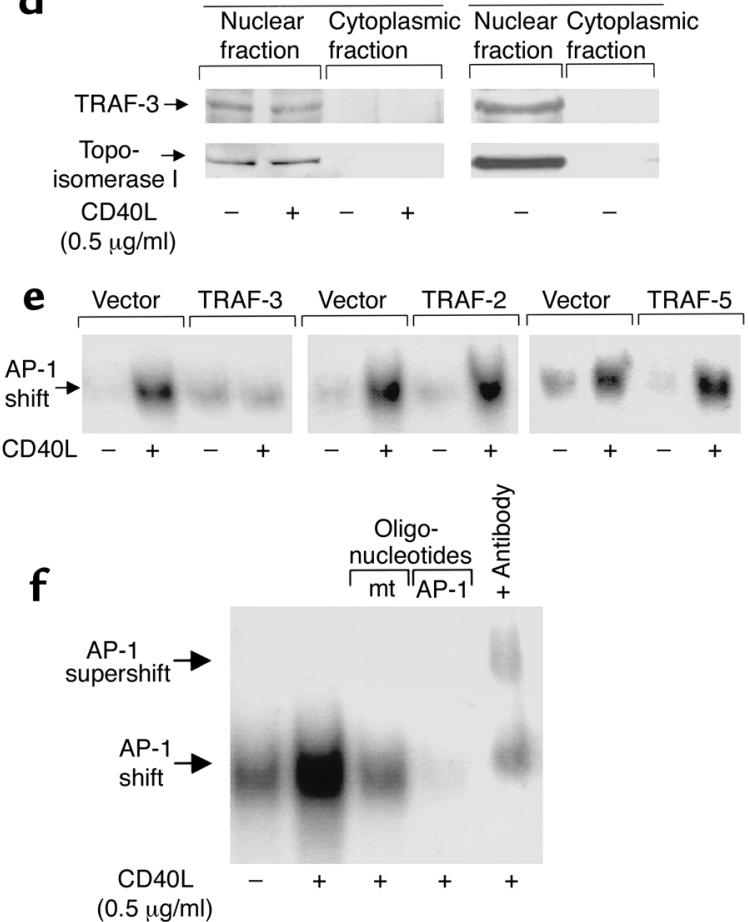

Figure 5

TRAF-3 blocks CD40L-induced AP-1 DNA binding activity. ( $\mathbf{a}$ and $\mathbf{b}$ ) 18 hours after transfection, HUVECs were incubated with CD40L, and phosphorylation of JNK (a, left panel), c-Jun (a, right panel), and ERK1/2 (b) was detected by Western blot analysis using phosphospecific antibodies. Blots were reprobed with actin or total ERK1/2 to confirm equal loading. Representative blots from three independent experiments are shown. (c) HUVECs were incubated with CD40L for 6 hours or exposed to shear stress for 18 hours. Cells were stained with antibodies against TRAF-2, TRAF-3, or TRAF-5, and secondary FITC-labeled anti-rabbit antibody (left column), followed by counterstaining with DAPI (middle column). The right column shows the overlay of both. $n=3-5$. (d) HUVECs or human cardiac microvascular endothelial cells (HMVECs) were incubated with CD40L for 6 hours. Nuclear and cytoplasmic proteins were isolated, and TRAF-3 expression was detected by Western blot analysis. Topoisomerase I was used as a nuclear marker protein. $n=3-5$. (e) HUVECs were transfected with TRAF-2, TRAF-3, TRAF-5, or control vector. HUVECs were stimulated with CD40L for 1 hour, and AP- 1 DNA binding activity was determined in nuclear extracts by EMSA using a ${ }^{32}$ P-labeled AP- 1 oligonucleotide probe. $n=3-6$. (f) Specificity of binding was examined by competition with 100-fold excess of unlabeled AP-1 oligonucleotides or mutated AP-1 oligonucleotides (mt). For supershift analysis, CD40-stimulated nuclear extracts were preincubated with an AP-1 antibody. $n=3-6$.

sclerotic lesions (9). The inhibition of CD40 signaling occurs in areas of the endothelial lining exposed to laminar shear stress. These areas are characteristically associated with atherosclerotic lesion-protected arterial geometries in vivo. The inhibition of CD40 signaling thereby may represent a mechanism that would explain the well-known atheroprotective effects of shear stress.

Beyond the regulation of lesion development, CD40 also triggers procoagulatory effector pathways that are involved in thrombus formation. Indeed, CD40L is significantly elevated in patients with acute coronary syndromes (30). Therefore, TRAF-3-mediated inhibition of tissue factor expression induced by
CD40L or activated platelets may additionally limit the procoagulatory activity of the endothelium, which covers the vessel wall and the plaque surface.

Exposure of endothelial cells to shear stress exerts profound effects on endothelial cell morphology and function. These effects are mediated by transcriptional changes in gene expression and posttranscriptional regulation of various signal transduction pathways (19, 31). A stepwise increase from static to flow conditions is known to induce an acute, short-term activation of proinflammatory pathways, including MCP- 1 and tissue factor expression (32-34). In contrast, long-term exposure of endothelial cells to shear, which more close- 
ly resembles the in vivo conditions, suppresses gene expression of MCP-1 (32). The present study extends these findings by demonstrating that preexposure of endothelial cells to shear stress for 12 hours prevents CD40L-induced MCP-1 and tissue factor expression.

The inhibitory effect of shear stress appears to rely on the expression of TRAF-3. However, overexpression of dominant-negative TRAF-3 or TRAF-3 antisense oligonucleotides (data not shown) only partially reversed the inhibitory effect of shear stress, suggesting that additional shear stress-induced signaling pathways can compensate for the loss of TRAF-3. Shear-induced increases in nitric oxide synthesis $(35,36)$ may interfere with AP-1 activation by inhibition of JNK caused by Snitrosylation (37), or by direct interference with AP-1 binding activity (38-40). However, inhibition of endothelial nitric oxide synthase by $\mathrm{N}^{\mathrm{G}}$-mono-methylL-arginine did not reverse the inhibitory effect of shear stress in the presence of dominant-negative TRAF- 3 (data not shown), implicating a nitric oxide-independent pathway. Another possibility might be that shear stress inhibits JNK activation through cross-talk among MAPK family members, as suggested recently by Surapisitchat and coworkers (41), who demonstrated that TNF- $\alpha$-induced JNK activation is inhibited by shear stress-induced activation of ERK1/2. Consistent with these findings, shear stress was also shown to block JNK activation via inhibition of the MAPK kinase kinase apoptosis signal-regulating kinase 1 (ASK-1) (42). Of note, both of these studies investigated shortterm effects of shear stress (10 minutes), whereas in the present study, cells were continuously preexposed to shear stress for 12 hours. This may explain why we did not find a direct inhibition of CD40-stimulated JNK activity, but an inhibition of the CD40-induced gene expression caused by increased expression of TRAF- 3 after long-term exposure of endothelial cells to flow. The remaining TRAF-3-independent inhibition of CD40L-induced endothelial activation by prolonged flow exposure requires further elucidation.

Although inhibition of TRAF- 3 only partially reversed the protective effect of shear stress, TRAF- 3 overexpression prevented CD40L-induced MCP-1 expression, suggesting that TRAF-3 is sufficient to block CD 40 signaling. TRAF-3 seems to interfere with CD40 signaling by inhibiting AP-1 DNA-binding activity. This is surprising, taking into account that a yeast two-hybrid screen identified TRAF-3 as a CD40 receptor-binding protein (27). However, immunohistochemistry and biochemical fractionation clearly revealed different localization of TRAF- 3 in endothelial cells than in NIH $3 \mathrm{~T} 3$ cells, with TRAF-3 being localized mainly in the nuclei of endothelial cells. In addition, no interaction of TRAF- 3 with the CD40 receptor was detectable in coimmunoprecipitation studies (data not shown). Interestingly, TRAF-3 is the only protein within the TRAF superfamily that contains a basic leucine zipper motif at amino acid position 287-342 (27). Given that proteins with a basic leucine zipper readily form homo- or heterodimers, and thereby provide a diversity of tran- scription factor complexes to inhibit or activate gene expression (29), one might speculate that TRAF- 3 acts by interacting with AP-1 complex formation.

Shear stress specifically upregulates TRAF- 3 expression, whereas TRAF- 2 and TRAF- 5 were not affected by exposure to shear stress. The question remains how shear stress increases TRAF- 3 expression. Shear stress can activate a variety of different transcription factors, including SP-1, AP-1 (c-Jun/c-Fos), and NF-KB (19, 43). However, TRAF-3 transcription was essentially unchanged after exposure to shear stress, as determined by nuclear run-on assay. These data suggest that shear stress stabilizes TRAF- 3 mRNA and thereby augments TRAF- 3 expression. Dissection of the molecular pathways involved in TRAF-3 stabilization requires further investigation.

In conclusion, the data of the present study suggest that selective upregulation of TRAF-3 by shear stress may provide a novel anti-inflammatory and anticoagulatory strategy to protect the endothelium.

\section{Acknowledgments}

We would like to thank Iris Henkel, Christiane Mildner-Rihm, Melanie Näher, and Susanne Ficus for expert technical assistance, and Beate Fiblthaler and Valerie Schini-Kerth (Department of Physiology, University of Frankfurt, Frankfurt, Germany) for technical support. This work was supported by the Deutsche Forschungsgemeinschaft (Di600/2-3).

1. Ross, R. 1999. Atherosclerosis: an inflammatory disease. N. Engl. J. Med. 340:115-126.

2. Gimbrone, M.A., Jr., Topper, J.N., Nagel, T., Anderson, K.R., and GarciaCardena, G. 2000. Endothelial dysfunction, hemodynamic forces, and atherogenesis. Ann. NY Acad. Sci. 902:230-240.

3. Dechanet, J., et al. 1997. CD40 ligand stimulates proinflammatory cytokine production by human endothelial cells. J. Immunol. 159:5640-5647.

4. Schonbeck, U., et al. 1997. Ligation of CD40 activates interleukin 1betaconverting enzyme (caspase-1) activity in vascular smooth muscle and endothelial cells and promotes elaboration of active interleukin 1beta. J. Biol. Chem. 272:19569-19574.

5. Slupsky, J.R., et al. 1998. Activated platelets induce tissue factor expression on human umbilical vein endothelial cells by ligation of CD40. Thromb. Haemost. 80:1008-1014.

6. Mach, F., et al. 1999. T lymphocytes induce endothelial cell matrix metalloproteinase expression by a CD40L-dependent mechanism: implications for tubule formation. Am. J. Pathol. 154:229-238.

7. Phipps, R.P. 2000. Atherosclerosis: the emerging role of inflammation and the CD40-CD40 ligand system. Proc. Natl. Acad. Sci. USA. 97:6930-6932.

8. Mach, F., et al. 1997. Functional CD40 ligand is expressed on human vascular endothelial cells, smooth muscle cells, and macrophages: implications for CD40-CD40 ligand signaling in atherosclerosis. Proc. Natl. Acad. Sci. USA. 94:1931-1936.

9. Mach, F., Schonbeck, U., Sukhova, G.K., Atkinson, E., and Libby, P. 1998. Reduction of atherosclerosis in mice by inhibition of CD40 signalling. Nature. 394:200-203.

10. Schonbeck, U., Sukhova, G.K., Shimizu, K., Mach, F., and Libby, P. 2000. Inhibition of CD40 signaling limits evolution of established atherosclerosis in mice. Proc. Natl. Acad. Sci. USA. 97:7458-7463.

11. Lutgens, E., et al. 1999. Requirement for CD154 in the progression of atherosclerosis. Nat. Med. 5:1313-1316.

12. Krzesz, R., Wagner, A.H., Cattaruzza, M., and Hecker, M. 1999. Cytokineinducible CD40 gene expression in vascular smooth muscle cells is mediated by nuclear factor kappaB and signal transducer and activation of transcription-1. FEBS Lett. 453:191-196.

13. Arch, R.H., Gedrich, R.W., and Thompson, C.B. 1998. Tumor necrosis factor receptor-associated factors (TRAFs) - a family of adapter proteins that regulates life and death. Genes Dev. 12:2821-2830. 
14. Rothe, M., Sarma, V., Dixit, V.M., and Goeddel, D.V. 1995. TRAF2-mediated activation of NF-kappa B by TNF receptor 2 and CD40. Science. 269:1424-1427.

15. Ishida, T.K., et al. 1996. TRAF5, a novel tumor necrosis factor receptorassociated factor family protein, mediates CD40 signaling. Proc. Natl. Acad. Sci. USA. 93:9437-9442.

16. Gimbrone, M.A., Nagel, T., and Topper, J.N. 1997. Biomechanical activation: an emerging paradigm in endothelial adhesion biology. J. Clin. Invest. 99:1809-1813.

17. Zarins, C.K., et al. 1983. Carotid bifurcation atherosclerosis. Quantitative correlation of plaque localization with flow velocity profiles and wall shear stress. Circ. Res. 53:502-514.

18. Traub, O., and Berk, B.C. 1998. Laminar shear stress: mechanisms by which endothelial cells transduce an atheroprotective force. Arterioscler. Thromb. Vasc. Biol. 18:677-685.

19. Chien, S., Li, S., and Shyy, Y.J. 1998. Effects of mechanical forces on signal transduction and gene expression in endothelial cells. Hypertension. 31:162-169.

20. Dimmeler, S., et al. 1999. Activation of nitric oxide synthase in endothelial cells via Akt-dependent phosphorylation. Nature. 399:601-605.

21. Clauss, M., et al. 1996. Synergistic induction of endothelial tissue factor by tumor necrosis factor and vascular endothelial growth factor: functional analysis of the tumor necrosis factor receptors. FEBS Lett. 390:334-338.

22. Urbich, C., Walter, D.H., Zeiher, A.M., and Dimmeler, S. 2000. Laminar shear stress upregulates integrin expression: role in endothelial cell adhesion and apoptosis. Circ. Res. 87:683-689.

23. Force, W.R., Cheung, T.C., and Ware, C.F. 1997. Dominant negative mutants of TRAF3 reveal an important role for the coiled coil domains in cell death signaling by the lymphotoxin-beta receptor. J. Biol. Chem. 272:30835-30840.

24. Gamper, C., et al. 2000. TRAF-3 interacts with p62 nucleoporin, a component of the nuclear pore central plug that binds classical NLS-containing import complexes. Mol. Immunol. 37:73-84.

25. Henn, V., et al. 1998. CD40 ligand on activated platelets triggers an inflammatory reaction of endothelial cells. Nature. 391:591-594.

26. VanArsdale, T.L., et al. 1997. Lymphotoxin-beta receptor signaling complex: role of tumor necrosis factor receptor-associated factor 3 recruitment in cell death and activation of nuclear factor kappaB. Proc. Natl. Acad. Sci. USA. 94:2460-2465.

27. Cheng, G., et al. 1995. Involvement of CRAF1, a relative of TRAF, in CD40 signaling. Science. 267:1494-1498.

28. Leo, E., et al. 1999. Differential requirements for tumor necrosis factor receptor-associated factor family proteins in CD40-mediated induction of NF-kappaB and Jun N-terminal kinase activation. J. Biol. Chem. 274:22414-22422.
29. Karin, M., Liu, Z., and Zandi, E. 1997. AP-1 function and regulation. Curr. Opin. Cell Biol. 9:240-246.

30. Aukrust, P et al 1999. Enhanced levels of soluble and membrane-bound CD40 ligand in patients with unstable angina. Possible reflection of T lymphocyte and platelet involvement in the pathogenesis of acute coronary syndromes. Circulation. 100:614-620.

31. Garcia-Cardena, G., Comander, J., Anderson, K.R., Blackman, B.R., and Gimbrone, M.A., Jr. 2001. Biomechanical activation of vascular endothelium as a determinant of its functional phenotype. Proc. Natl. Acad. Sci. USA. 98:4478-4485

32. Shyy, Y.J., Hsieh, H.J., Usami, S., and Chien, S. 1994. Fluid shear stress induces a biphasic response of human monocyte chemotactic protein 1 gene expression in vascular endothelium. Proc. Natl. Acad. Sci. USA. 91:4678-4682.

33. Shyy, J.Y., et al. 1995. The cis-acting phorbol ester " 12 -O-tetradecanoylphorbol 13-acetate"-responsive element is involved in shear stressinduced monocyte chemotactic protein 1 gene expression. Proc. Natl. Acad. Sci. USA. 92:8069-8073.

34. Lin, M.C., et al. 1997. Shear stress induction of the tissue factor gene. J. Clin. Invest. 99:737-744.

35. Noris, M., et al. 1995. Nitric oxide synthesis by cultured endothelial cells is modulated by flow conditions. Circ. Res. 76:536-543.

36. Uematsu, M., et al. 1995. Regulation of endothelial cell nitric oxide synthase mRNA expression by shear stress. Am. J. Physiol. 269:C1371-C1375

37. Park, H.S., Huh, S.H., Kim, M.S., Lee, S.H., and Choi, E.J. 2000. Nitric oxide negatively regulates c-Jun N-terminal kinase/stress-activated protein kinase by means of S-nitrosylation. Proc. Natl. Acad. Sci. USA. 97:14382-14387.

38. Tabuchi, A., Sano, K., Oh, E., Tsuchiya, T., and Tsuda, M. 1994. Modulation of AP-1 activity by nitric oxide (NO) in vitro: NO-mediated modulation of AP-1. FEBS Lett. 351:123-127.

39. Nikitovic, D., Holmgren, A., and Spyrou, G. 1998. Inhibition of AP-1 DNA binding by nitric oxide involving conserved cysteine residues in Jun and Fos. Biochem. Biophys. Res. Commun. 242:109-112.

40. Marshall, H.E., Merchant, K., and Stamler, J.S. 2000. Nitrosation and oxidation in the regulation of gene expression. FASEB J. 14:1889-1900.

41. Surapisitchat, J., et al. 2001. Fluid shear stress inhibits TNF-alpha activation of JNK but not ERK1/2 or p38 in human umbilical vein endothelial cells: inhibitory crosstalk among MAPK family members. Proc. Natl. Acad. Sci. USA. 98:6476-6481.

42. Liu, Y., Yin, G., Surapisitchat, J., Berk, B.C., and Min, W. 2001. Laminar flow inhibits TNF-induced ASK1 activation by preventing dissociation of ASK1 from its inhibitor 14-3-3. J. Clin. Invest. 107:917-923.

43. Shyy, J.Y., and Chien, S. 1997. Role of integrins in cellular responses to mechanical stress and adhesion. Curr. Opin. Cell Biol. 9:707-713. 